\title{
POSTERIOR MAXILLARY RIDGE AUGMENTATION WITH SINUS LIFT USING MINERALIZED PLASMATIC MATRIX VERSUS AUTOGENOUS BONE GRAFT (A RANDOMIZED CLINICAL TRIAL COMPARATIVE STUDY)
}

\author{
Refaat Ragab Abou-Ellill, Ragia Mohamed Mounir* and Ahmed Tarek Elsharkawy***
}

\begin{abstract}
Aim: The aim of this study was to assess the effect of augmentation using mineralized plasmatic matrix versus autogenous bone graft on bone formation and implant stability.

Methodology: Twenty Implants were inserted in the maxillary premolar and molar region after augmentation of 8 maxillary sinuses in 6 patients, divided into 2 groups, study group received MPM as a graft, while the control group received autogenous particulate bone.

Results: There was no statistically significant difference between both groups regarding implant stability immediately after implant insertion; however, after 5-month stability in the study group was statistically significantly higher than the control group. The mean change in bone height after 4 months in the study group was statistically significantly higher than in the control group.

Conclusion: The use of MPM in osseous regeneration and implant therapy has a positive impact on the outcome of the grafting surgery when used for open sinus lifting.
\end{abstract}

KEYWORDS: sinus lifting, MPM, sticky bone, maxillary augmentation, open sinus.

\section{INTRODUCTION}

The goal of modern dentistry is to restore the alveolar ridges and teeth to normal contour, function, aesthetics, speech, and health. The more teeth a patient is missing, the more challenging this task becomes. One of the challenges with implant placement is an unfavourable local condition of the alveolar ridge due to atrophy, which may cause insufficient bone volume in the horizontal and/or vertical dimensions ${ }^{1}$.

Proper bone-augmentation strategies incorporated within a treatment plan can enable the clinician to avoid such difficulties. Common bone regeneration indications include extraction sockets preservation,

\footnotetext{
* Master Degree in Oral and Maxillofacial Surgery

** Professor of Oral and Maxillofacial Surgery Cairo University

*** Assistant Professor of Oral and Maxillofacial Surgery Cairo University
} 
horizontally and vertically atrophic ridges, implantassociated defects, and other anatomical structures that may inhibit proper implant positioning. Many options for the treatment of alveolar ridge defects are available, including variable surgical techniques as well as different bone grafting options ${ }^{2}$. The technique and type of material used depend on the size and location of the defect ${ }^{3,4}$.

Platelet Rich Plasma (PRP) and Platelet Rich Fibrin (PRF) are inexpensive ways to obtain many GFs in physiological proportion and have already been largely applied as a carrier of GFs in different fields of medicine due to their property of favouring tissue healing even in tissues with low healing potential ${ }^{5,6}$. These techniques are based on the idea of the concentration of platelets for reuse as a drug in some serious diseases ${ }^{7-9}$. The platelet's release of cytokines can stimulate the colonization and proliferation of other cells which are important for the repair or regeneration process. Both products were used in dental implant therapy either to accelerate healing or to help to regenerate bone where needed. Several studies have reported their use in combination with autologous bone to improve bone handling and implant integration ${ }^{10}$.

A new technique has been recently developed based also on the concentration of platelets called MPM (Mineralized Plasmatic Matrix) sometimes referred to as (Sticky bone). On clinical evaluation comparing both PRF and MPM, MPM being sticky and rich in Growth factors offered better clinical and biological properties to the sites of the graft in the peri-implant sites ${ }^{11}$.

\section{AIM OF THE STUDY}

The aim of this study was to assess the effect of posterior maxillary ridge augmentation using mineralized plasmatic matrix versus autogenous bone graft on implant body stability and bone volume.

\section{MATERIALS AND METHODS}

The trial design of this study was a prospective, randomized, double-blinded clinical design. Patients were selected from those attending the outpatient department, faculty of dentistry, Cairo University, seeking restoration of missing posterior maxillary teeth.

\section{The inclusion criteria were}

Age 20-55 years, Males or females who were physically able to tolerate surgical and restorative procedures and having posterior maxillary ridge less than $6 \mathrm{~mm}$ height and more than $4.5 \mathrm{~mm}$ width.

\section{The exclusion criteria were}

Smokers, pregnant females, patients with a systemic disorder affecting bone healing, presence of any pathosis in the peri-implant site, history of oral radiotherapy, prolonged use of steroids and patients with psychological disorders.

\section{Sample size}

A total sample size of 20 (implants) used for 8 sinuses in 6 patients were sufficient with power $80 \%$ and $5 \%$ significance level. The sample size was calculated by the SPSS program.

\section{Randomization}

Patients were divided into 2 groups; the control group was the group of participants who received autogenous bone, while the intervention group was the group of participants where MPM was applied for open sinus lifting. Patients were randomized into two groups A and B with 10 implants in 4 sinuses for 3 patients in each group.

\section{I) Clinical Examination}

Clinical examination of each patient included inspection and palpation of surgical sites, evaluation of inter-ridge space, alveolar ridge contour and width, condition of the maxillary posterior teeth, and surrounding alveolar bone. 


\section{II) Radiographic examination}

A preoperative panoramic radiograph was taken for each patient for preliminary evaluation of the residual alveolar ridge and to detect any remaining roots or pathosis. Preoperative Conebeam computed tomography (CBCT) scans were taken for selected patients to examine the sinus for pathologies, plan implant sites, and residual alveolar bone height (Fig, 1).

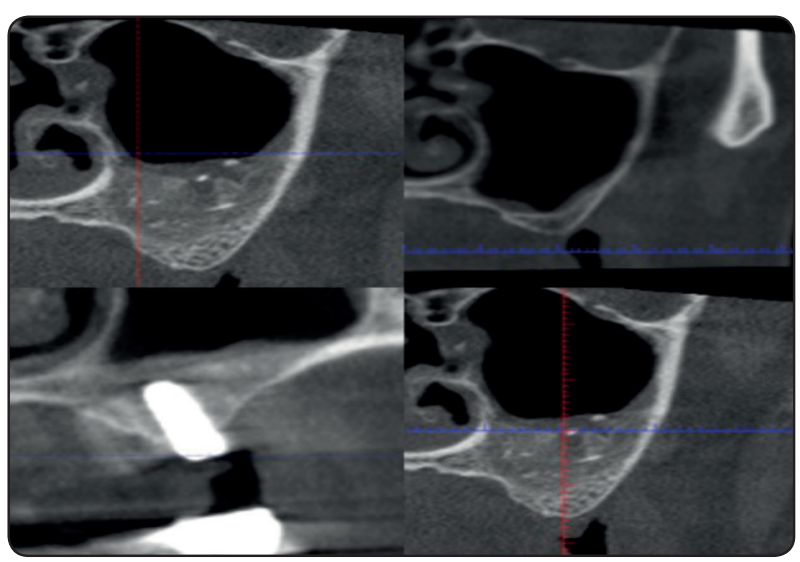

Fig. (1) Photo radiograph of bone gain before, after grafting and post implant (study group) in molar region

\section{III) Laboratory investigations}

Each selected patient was asked for a complete blood picture, coagulation profile (including INR, PT, PTT and BT), and random blood glucose, to rule out any hidden systemic condition that may affect bone and implant healing.

\section{Surgical steps (control group)}

First stage surgery: Each patient received local anaesthesia by infiltration in the Recipient site and bilateral mental nerve block for the donor site, using Articaine 4\% with 1:100000 adrenaline*. After confirming the success of profound local anaesthesia, an incision was made using blade no.
15 , and a pyramidal flap for the recipient site was reflected along the ridge using a sharp periosteal elevator, and then retracted using a Minnesota retractor.

Maxillary sinus floor elevation using a lateral window technique was performed for each patient using magnetic burs under copious irrigation, followed by sinus membrane lifting using special sinus lifting elevators.

Chin Donor site surgery: The incision was performed $5 \mathrm{~mm}$ below the mucogingival junction and through the mentalis muscle using number 15 scalpels in 2 layers. Dissection is performed through mentalis muscle to expose bone of the chin. By using a large trephine bur of circumference $3 \mathrm{~mm}$, or chisel and mallet to obtain a bone cylinder of approximately $5 \mathrm{~mm}$ length. The donor site was filled with gel foam, Sutures were performed along both layers, mentalis muscle reattachment by an absorbable vicryl suture 000, and the mucosal layer was sutured using the same material or nonabsorbable 000 silk types. By using a bone mill, the cylindrical piece of bone obtained from the chin was crushed to reach homogenous particulates, these particulates were mixed with drops of saline to ease the manipulation process, then applied to the lifted sinus floor. Edges of recipient site were approximated and Sutured using interrupted and horizontal mattress with vicryl 000 suture. Iliac graft was obtained in cases where a large amount of bone was required for bilateral sinus lifting.

Each patient received Augmentin** (1 gm combination of amoxicillin and clavulanic acid) every 12 hrs for 5 days and analgesic and antiinflammatory $* * *$ (Ibuprofen $600 \mathrm{mg}$ ) every 8 hrs. for 3 days and a **** chlorhexidine-based mouthwash every $8 \mathrm{hrs}$ for 15 days and decongestant

\footnotetext{
* "Inibsa" Ubistesin forte 4\% Articain (Spain)

** Augmentin; GlaxoSmithKline, Munich, Germany.

*** Ibuprofen; Abbott international Egypt, Kahira pharmaceutical.

**** Anti septol has been manufactured and distributed by Johnson \& Johnson
} 
nasal drop * (Afrin) twice daily after the surgical procedure.

Second stage surgery: A cone-beam CT was performed to measure bone height and plan implant dimensions after 4 months. At the time of implant placement, Core biopsy specimens were obtained from the planned implant sites for histological evaluation. Core biopsy was saved in $10 \%$ formaldehyde solution for histomorphometry. Sequential socket drilling and implant placement was done using ** Mode implants 3.7 and 4.2 diameters.

Study Group: Same procedure was made for the surgical phase of the study group as in the control group, except that bone was mixed with the supernatant fluid obtained from blood centrifuged in a plain tube for 5 minutes at $2700 \mathrm{rpm}$, till homogenous sticky bone was obtained (Fig. 2,3).

Smart beg was applied to implant hex to measure and record fixture primary stability in ISQ units precisely and objectively. After 5 months (time of implant loading) ostell readings were recorded again for comparison.

Post-operative follow-up: Patients were assessed on regular intervals: 72 hours, 1 week, 2

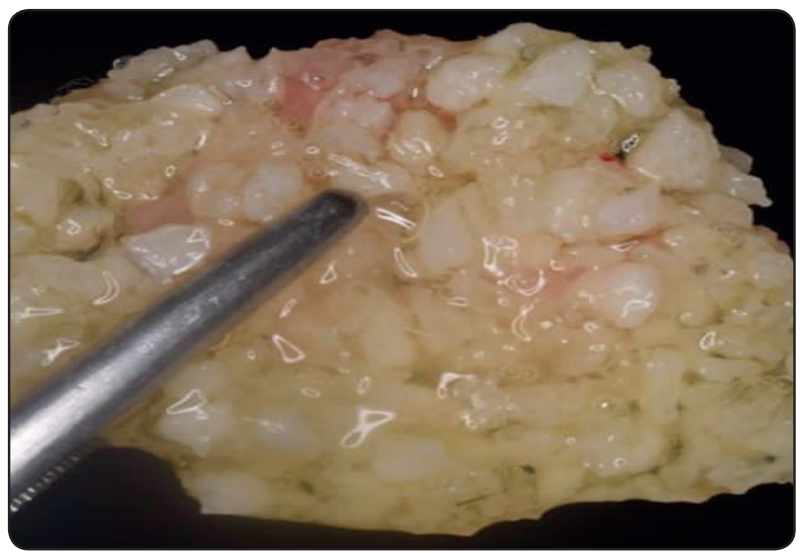

Fig. (2): Photograph showing sticky bone obtained (MPM).

\footnotetext{
* Afrin; Merck\& Co., Inc, USA.

** Implant type: Mode, Istanbul, turkey.

*** SPSS, Inc., Chicago, IL, USA.
}

weeks, 1 month, and monthly till 9 months. Patients were assessed objectively and subjectively for any complications such as bleeding, hematoma, infection, persistent pain, implant or bone graft exposure or loss, altered sensation of lower lip or chin or lower teeth at the donor site.

Radiographic follow-up: Postoperative CBCT was taken for the patients at 4 months. Readings of the radiographic assessment of linear measurements of bone height were calculated, compared with preoperative values, and statistically analysed.

Statistical Analysis: All data were collected and tabulated. Statistical analysis was performed by IBM and Statistical Package for Social Science*** (SPSS) version 20 for windows. Data were collected for each patient including base line data, Osstell scores, and bone height in millimeters. Parametric data were presented by mean and standard deviation (mean \pm SD) and categorical data were presented as frequency (number of cases) and percentage. Independent t-test $(\mathrm{T})$ was used to compare between groups at each interval. The paired student " $\mathrm{t}$ " test was used to compare implant stability and bone height at each interval in the same group. The significance level was set at $\mathrm{P} \leq 0.05$.

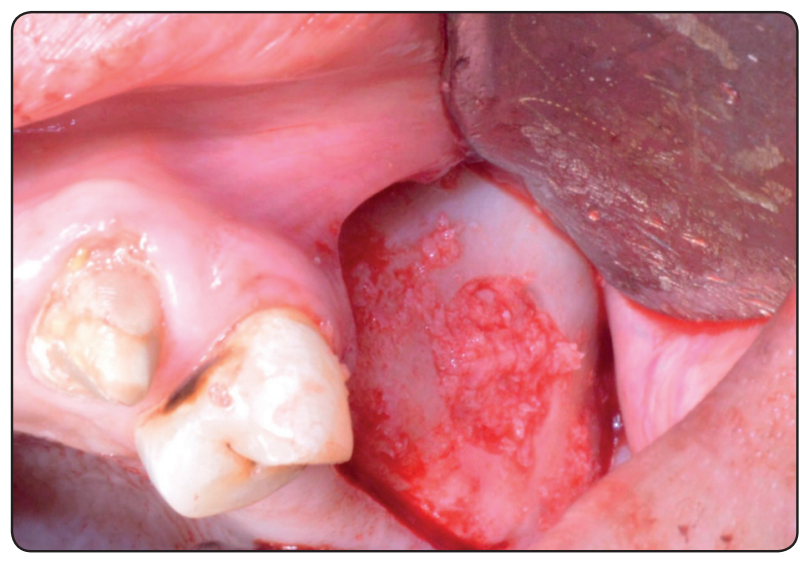

Fig. (3): Photograph showing application of MPM graft to the sinus in control group. 


\section{RESULTS}

The selected patients were 4 females and 2 males, 12 implants were inserted in females and 8 implants in males, their age ranged from $29-45$ years with a mean age (39).

Clinical results: The healing of all flaps was uneventful. After 5 months of stage 2 surgery patients were recalled for clinical assessment and for designing the prosthetic part (stage 3 surgery), all patients received implant-supported Porcelain fused to metal cemented crowns.

\section{Implant stability results:}

TABLE (1): Paired student " $\mathrm{t}$ " test comparing stability in control group immediately (implant insertion) and after 5 months.

\begin{tabular}{|c|c|c|c|c|c|}
\hline \multicolumn{2}{|c|}{ Immediate } & \multicolumn{2}{|c|}{ After 5 Months } & \multirow{2}{*}{ "t” } & \multirow{2}{*}{ Probability } \\
\cline { 1 - 4 } Mean & St Dev & Mean & St Dev & & \\
\hline 46.25 & 3.97 & 62.65 & 5.33 & 12.881 & 0.0000 \\
\hline
\end{tabular}

There was a statistically significant increase in stability after 5 months

TABLE (2): Paired student " $t$ " test comparing stability in study group immediately (implant insertion) and after 5 months.

\begin{tabular}{|c|c|c|c|c|c|}
\hline \multicolumn{2}{|c|}{ Immediate } & \multicolumn{2}{c|}{ After 5 Months } & \multirow{2}{*}{ “t” } & \multirow{2}{*}{ Probability } \\
\cline { 1 - 4 } Mean & St Dev & Mean & St Dev & & \\
\hline 49.85 & 6.25 & 67.2 & 5.49 & 10.602 & 0.0000 \\
\hline
\end{tabular}

There was a statistically significant increase in stability after 5 months

TABLE (3): Independent student " $t$ " test comparing the change in stability in both groups after 5 months

\begin{tabular}{|c|c|c|c|c|c|}
\hline \multicolumn{2}{|c|}{ Control } & \multicolumn{2}{c|}{ Study } & \multirow{2}{*}{ "t” } & \multirow{2}{*}{ Probability } \\
\cline { 1 - 4 } Mean & St Dev & Mean & St Dev & & \\
\hline 16.4 & 4.03 & 17.35 & 5.17 & 0.458 & 0.326 NS \\
\hline
\end{tabular}

There was no statistically significant difference between changes of stability in both groups after 5 months

TABLE (4): Independent student " $t$ " comparing both groups immediately (implant insertion) and after 5 months.

\begin{tabular}{|c|c|c|c|c|c|c|}
\hline \multirow{2}{*}{ Stability } & \multicolumn{2}{|c|}{ Control } & \multicolumn{2}{|c|}{ Study } & \multirow{2}{*}{ "t" } & \multirow{2}{*}{ Probability } \\
\cline { 2 - 6 } & Mean & $\begin{array}{c}\text { St } \\
\text { Dev }\end{array}$ & Mean & $\begin{array}{c}\text { St } \\
\text { Dev }\end{array}$ & & \\
\hline Immediate & 46.25 & 3.97 & 49.85 & 6.25 & 1.539 & $0.071 \mathrm{NS}$ \\
\hline $\begin{array}{c}\text { After 5 } \\
\text { Months }\end{array}$ & 62.65 & 5.33 & 67.2 & 5.49 & 1.879 & $0.038 *$ \\
\hline
\end{tabular}

Regarding implant stability, there was no statistically significant difference between both groups immediately after implant insertion, while after 5 months the implant stability in the study group was statistically significantly higher than the control group.

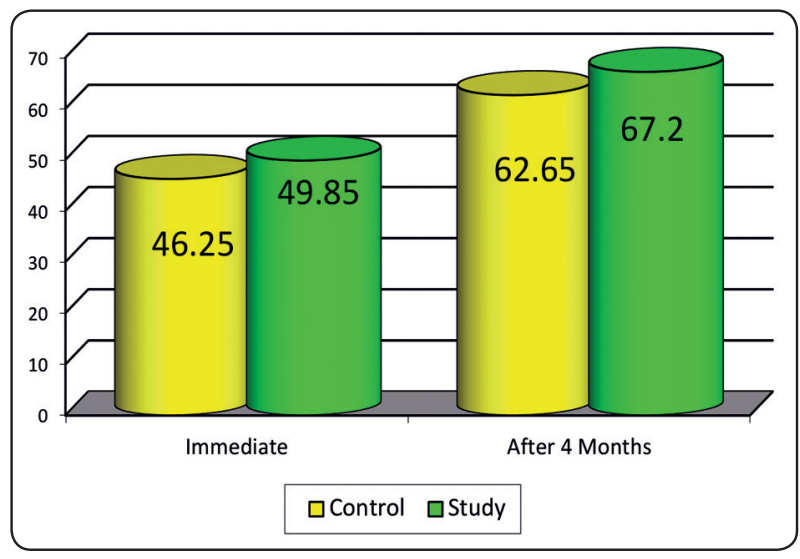

Fig. (4): Mean values of implant stability in both groups immediately and after 5 months.

\section{Radiographic results:}

TABLE (5): Paired student " $t$ " test comparing bone height $(\mathrm{mm})$ in the control group Preoperative and after 4 months.

\begin{tabular}{|c|c|c|c|c|c|}
\hline \multicolumn{2}{|c|}{ Pre-operative } & \multicolumn{2}{|c|}{ After 9 Months } & \multirow{2}{*}{ " $\mathrm{t}$ ” } & \multirow{2}{*}{ Probability } \\
\cline { 1 - 4 } Mean & St Dev & Mean & St Dev & & \\
\hline 3.97 & 0.84 & 9.69 & 0.84 & 36.947 & 0.0000 \\
\hline
\end{tabular}


There was a statistically significant increase in bone height $(\mathrm{mm})$ after 4 months.

TABLE (6): Paired student " $t$ " test comparing bone height $(\mathrm{mm})$ Pre-operative and after 4 months (study group)

\begin{tabular}{|c|c|c|c|c|c|}
\hline \multicolumn{2}{|c|}{ Pre-operative } & \multicolumn{2}{c|}{ After 9 Months } & \multirow{2}{*}{ “t” } & \multirow{2}{*}{ Probability } \\
\cline { 1 - 3 } Mean & St Dev & Mean & St Dev & & \\
\hline 4.63 & 1.40 & 11.45 & 1.96 & 15.355 & 0.0000 \\
\hline
\end{tabular}

There was a statistically significant increase in bone height $(\mathrm{mm})$ after 4 months

TABLE (7): Independent student " $t$ " test comparing the change in bone height $(\mathrm{mm})$ in both groups after 4 months

\begin{tabular}{|c|c|c|c|c|c|}
\hline \multicolumn{2}{|c|}{ Control } & \multicolumn{2}{|l|}{ Study } & \multirow{2}{*}{ "t" } & \multirow{2}{*}{ Probability } \\
\hline Mean & St Dev & Mean & St Dev & & \\
\hline 5.72 & 0.49 & 6.83 & 1.41 & 2.351 & 0.015 \\
\hline
\end{tabular}

The mean change in bone height after 4 months in the study group was statistically significantly higher than in the control group.

TABLE (8): Independent student " $t$ " comparing the bone height of both groups' Pre-operative and after 4 months.

\begin{tabular}{|c|c|c|c|c|c|c|}
\hline \multirow{2}{*}{$\begin{array}{c}\text { Bone } \\
\text { Height }\end{array}$} & \multicolumn{2}{|c|}{ Control } & \multicolumn{2}{|c|}{ Study } & \multirow{2}{*}{ "t" } & Probability \\
\cline { 2 - 5 } & Mean & $\begin{array}{c}\text { St } \\
\text { Dev }\end{array}$ & Mean & $\begin{array}{c}\text { St } \\
\text { Dev }\end{array}$ & & \\
\hline $\begin{array}{c}\text { Pre- } \\
\text { operative }\end{array}$ & 3.97 & 0.84 & 4.63 & 1.40 & 1.266 & $0.111 \mathrm{NS}$ \\
\hline $\begin{array}{c}\text { After 9 } \\
\text { Months }\end{array}$ & 9.69 & 0.84 & 11.45 & 1.96 & 2.620 & $0.009 *$ \\
\hline
\end{tabular}

Regarding bone height, there was no statistically significant difference between both groups immediately after implant insertion, while after 4 months the implant stability in the study group was statistically significantly higher than the control group.

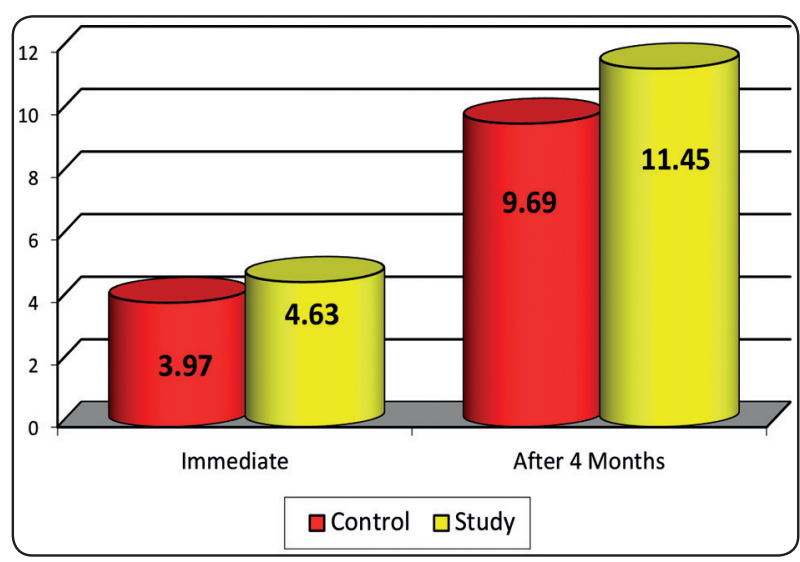

Fig (5): Mean values of bone height in both groups immediately and after 4 months.

\section{DISCUSSION}

Insufficient alveolar ridge height is often related to the proximity of the implant site to other anatomical structures, i.e. the maxillary sinus or the mandibular canal. Particularly in the posterior maxilla, lack of bone volume between the crest and the maxillary sinus is problematic which might cost the procedure of implant placement in the region extreme difficulty. To achieve optimal treatment outcomes with dental implants, sufficient bone should be available to support and stabilize them. Reconstruction of the alveolar bone through a variety of regenerative surgical procedures had become predictable where autogenous, allogenic, synthetic, and tissue-engineered bone grafts can be successfully used ${ }^{12,13,14}$.

In our study, autogenous bone grafts were used as they are the 'gold standard' in reconstructing small bone defects and are considered to be qualitatively superior to other types of grafts. The success rates in the autogenous bone graft are from $73.8 \%$ to $100 \%$. Autogenous bone grafts were taken from the chin or the iliac crest of the same patient have been the standard for alveolar reconstruction, specifically, due to their osteogenic, osteoconductive, osteoinductive, and lack of immunogenic properties; which will enhance graft consolidation, help maintain graft morphology and possibly improve long term survival ${ }^{15,16}$. 
In this study cortico-cancellous autogenous particulate bone graft was used as a grafting material in the control group. Several studies demonstrated the effectiveness of particulate autograft. The use of the particulate bone graft in implant dentistry has its limits. It is indicated in limited cases where the particulates are stable and cannot move. To secure the stability of the particles, membranes are used to hold the particles together and to stop the leak of the graft ${ }^{17}$.

MPM was chosen primarily because it is an autologous blood product highly concentrated in platelets and fibrin mixed with the mineral phase of bone graft forming a homogeneous single component, which is compact and stable, containing the graft, the dense fibrin network where the fibrin can become bound to bone particles, and the growth factors promoting healing. This procedure allows linking all the particulates together in one product. During manipulation, the retention in the fibrous mesh of the bone fragments or the grafting material conserves its cohesion and avoids its departure away from the recipient bed which may contribute to the increased bone volume gained and allow us to avoid the use of membranes ${ }^{18,19}$.

Other disadvantages of autogenous bone grafts are having a secondary surgical site required, another potential location for postoperative pain, and complications structural compromise at the donor site. MPM is an evolution of PRP which is an autologous modification of fibrin glue and is used to deliver the growth factors in high concentration to the bone site. These growth factors (PDGF and TGF- $\beta$ ) accelerate post-surgical healing, bone augmentation, and improve soft tissue texture. One of the highest concentrations of PDGF and TGF- $\beta$ in in the body is found within the blood platelets. This advantage may allow MPM obtained from allogenic bone or xenograft to obtain osteoinductive properties of autogenous bone and save future patients from donor site complications ${ }^{20}$.
In our study, there was a statistically significant difference between the 2 groups in favor of the MPM group regarding the gained bone height. Therefore, the use of MPM in implant therapy has a great impact on the outcome of the grafting surgery because it enhances the transport of the material by securing its implementation. This stability of the graft opened a new age of the use of particulate bone grafts ${ }^{21}$.

In addition to that, Perissé et al (2011) revealed the presence of monocytes inside the MPM biopsies ${ }^{19}$. Monocytes are very important in bone formation as they allow regulation of production of BMPs which are - according to a systematic review by Simpson et al 2006 - highly important proteins in the induction of bone production, in addition to their antibacterial action ${ }^{21}$.

In this study Osstell was used to assess implant stability. Implant stability is an indirect indication of Osseo integration. It is a measure of the clinical immobility of an implant. Secure primary stability leads to predictable secondary stability. Secondary stability has been shown to begin to increase at four weeks after implant placement. At this time point, the lowest implant stability is expected. Therefore, the original Bran mark protocol suggested a 3 to 6-month non-loaded healing period to achieve adequate stability before functional loading. Various methods have been introduced in order to evaluate implant stability. Recently, resonance frequency analysis (RFA), a non-invasive method developed by the study of Meredith, has been the most accepted technique ${ }^{22}$.

\section{CONCLUSION}

The results suggest that both techniques can be used to improve implant stability. The uses of MPM in osseous regeneration and implant therapy have a positive impact on the outcome of the grafting surgery. It eases and enhances the delivery and homogenization of the grafting materials, increase bone quality and quantity. 


\section{REFERENCES}

1. Misch CE, Perel ML, Wang HL et al: Implant Success, Survival, and Failure: The International Congress of Oral Implantologists (ICOI) Pisa Consensus Conference. Implant Dent. 2008; 17(1):5-15. 2.

2. Fugazzotto PA: GBR using bovine bone matrix and resorbable and nonresorbable membranes. Part 2: Clinical results. Int $\mathrm{J}$ Periodontics Restorative Dent. 2003;23(6):599-605.

3. Herford AS, Nguyen K: Complex Bone Augmentation in Alveolar Ridge Defects. Oral Maxillofac Surg Clin North Am. 2015;27(2): 227-44.

4. Vignoletti F, Matesanz P, Rodrigo D: Surgical protocols for ridge preservation after tooth extraction. A systematic review. Clin Oral Implants Res. 2012; 23:22-38.

5. Wang W, Ouyang Y, Poh CK: Orthopedic implant technology: biomaterials from past to future. Ann Acad Med Singapore. 2011; 40(5): 237-44.

6. Liu Y, de Groot K, Hunziker EB: BMP-2 liberated from biomimetic implant coatings induces and sustains direct ossification in an ectopic rat model. Bone. 2005;36(5): 745-57.

7. Sezgin Y, Uraz A, Taner IL, et al: Effects of plateletrich fibrin on healing of intra-bony defects treated with anorganic bovine bone mineral. Braz Oral Res. 2017; 31(1): e15.

8. Simonpieri A, Del Corso M, Sammartino G, et al: The Relevance of Choukroun's Platelet-Rich Fibrin and Metronidazole During Complex Maxillary Rehabilitation Using Bone Allograft. Part I: A New Grafting Protocol. Implant Dent. 2009; 18(2): 102-11.

9. Kotsakis G, Chrepa V, Katta S: Practical Application of the Newly Introduced Natural Bone Regeneration (NBR) Concept Utilizing Alloplastic Putty. Int J Oral ImplantolClin Res. 2011; 2(3): 145-9.

10. Roffi A, Filardo G, Kon E, Marcacci M: Does PRP enhance bone integration with grafts, graft substitutes, or implants? A systematic review.2013; 14: 330.

11. Périssé J: From PRF to mineralized plasma matrices in implantology. 59th Congress of the SFMBCB 2012; 02010.
12. Motamedian SR, Khojaste M, Khojasteh A. Success rate of implants placed in autogenous bone blocks versus allogenic bone blocks: A systematic literature review. Ann Maxillofacial Surg. 2016;6(1):78-90.

13. Khojasteh A, Behnia H, Hosseini FS, et al: The effect of PCL-TCP scaffold loaded with mesenchymal stem cells on vertical bone augmentation in dog mandible: A preliminary report. J Biomed Mater Res Part B Appl Biomatter. 2013;101B (5): 848-54.

14. Jafari an M, Eslaminejad MB, Khojasteh A, et al: Marrow-derived mesenchymal stem cells-directed bone regeneration in the dog mandible: a comparison between biphasic calcium phosphate and natural bone mineral. Oral Surgery, Oral Med Oral Pathol Oral Radial Endodontology. 2008;105(5): e14-e24.

15. El Moheb M,Al-zarea B, Sghaireen MG, et al: Mineralized Plasmatic Matrix to Enhance the Bone Grafting Technique. hard tissue bio. 2017; 26(3): 289-92.

16. Rogers GF, Greene AK. Autogenous Bone Graft. J Craniofacial Surg. 2012;23(1): 323-7.

17. Khojasteh A, Soheil far S, Mohajerani H, et al: The effectiveness of barrier membranes on bone regeneration in localized bony defects: a systematic review. Int J Oral Maxillofacial Implants. 2013; 28(4): 1076-89.

18. Nadon F, Chaput B, Peruses J, et al: Interest of Mineralized Plasmatic Matrix in Secondary Autogenous Bone Graft for the Treatment of Alveolar Clefts. J Craniofacial Surg. 2015;26(7): 2148-51.

19. Perissé J, Bouzekri-Alami H, Mazzoni-Vassalakyl L, et al: Clinical and histological aspect of Mineralized Plasmatic Matrices (MPM): new FRP protocol I Dental surgeons in France. Publication Letter de la Stomatology 53. 2012.

20. Albanese A, Licata ME, Palazzi B, et al: Platelet-rich plasma (PRP) in dental and oral surgery: from the wound healing to bone regeneration. Immune Ageing. 2013;10(1):23.

21. Simpson A, Mills L, Noble B. The role of growth factors and related agents in accelerating fracture healing. 2006;88(6): 701-5.

22. Atsu mi M, Park S-H, Wang H-L. Methods Used to Assess Implant Stability: Current Status. Int J Oral Maxillofacial Implants. 2007; 22(5): 743-54. 